\title{
Triage Accuracy and Its Association with Patient Factors Using Emergency Severity Index: Findings from United Arab Emirates
}

This article was published in the following Dove Press journal: Open Access Emergency Medicine

\author{
Yousif AISerkal' \\ Kalthoom AlBlooshi \\ Sumaya AlBlooshi ${ }^{3}$ \\ Yasir Khan (iD) ${ }^{4}$ \\ Sadaf A Naqvi (iD) 4 \\ Colin Fincham (D) \\ Noor AIMehiri ${ }^{2}$
}

'Hospital Sector, Ministry of Health and Prevention, Dubai, United Arab Emirates; ${ }^{2}$ Hospital Department, Ministry of Health and Prevention, Dubai, United Arab Emirates; ${ }^{3}$ Nursing Department, Ministry of Health and Prevention, Dubai, United Arab Emirates; ${ }^{4}$ Cerner Middle East, Dubai, United Arab Emirates
Correspondence: Sadaf A Naqvi

Email sadaf.naqvi@cerner.com
Introduction: The Ministry of Health and Prevention of the UAE acquired an electronic medical record system (Wareed) through which they incorporated the Emergency Severity Index as the standard triaging tool. This raised the need to review population dynamics and the accuracy of triage performed by the health-care providers utilizing the tool.

Objective: This research aimed to study demographics and dynamics of the population presenting to emergency departments (EDs) during 2018, evaluate the accuracy of triage assessment using comparative analysis techniques, and determine relationships between patient factors (severity of illness, age-group) and the accuracy of triage.

Methods: This was an observational study that aimed to ascertain findings from ED data over 1 year (January 2018-December 2018) and explore factors associated with reduced accuracy in acuity assignment. We employed comparative analysis to measure the level of agreement between standard guidelines and local findings.

Results: A total of 576,154 patients visited EDs in 2018, of which 54.4\% were male. A statistically significant increase in length of stay with increasing severity of illness was observed (Kruskal-Wallis test). Overall triage accuracy was $41.6 \%$, with a positive association with increasing severity of illness. We found a positive association between severity of illness and accuracy of triage (OR $0.14, p=0$ ). We also found on logistic regression that the age-group 11-20 years had the highest probability of accurate triage acuity $\left(R^{2}=0.41, p=0\right)$.

Conclusion: Conducted on a very large data set from the UAE, our study reflects upon population dynamics and triage accuracy distribution among different variables. This study paves the way for further in-depth analysis of factors that may impact triage accuracy within EDs, and utilizing a similar approach it can be replicated in other settings as well.

Keywords: Emergency Severity Index, ESI, triage, electronic medical record

\section{Introduction}

Emergency departments (EDs) are unique in their operation from the rest of the hospital, as they operate around the clock with heavy, unscheduled patient attendance and high turnover. ${ }^{1,2}$ Patients range from critical cases who require immediate livesaving interventions to those who are relatively stable. It lies with the ED to provide care to all patients based on the level of urgency and criticality of their illness. To make this process more structured and standardized, the triage pathway has been established. It is a process usually led by a trained clinician (nurse or physician), who at the time of patient's presentation quickly and accurately categorizes the patient 
according to their care needs. ${ }^{1}$ This leads to an efficient mechanism of prioritizing patients based on triage levels.

There are a number of triaging algorithms to support this process. The entire process should be completed swiftly, and should not itself become a cause for delay in the overall process. Since the care is delivered based on a level of urgency assumed by the triage clinician, inaccurate judgment can deprive some urgent cases of timely care and over-triaging can lead to unnecessary consumption of resources by patients that were less urgent. ${ }^{3,4}$ This calls for a system with high validity and reliability. ${ }^{5}$ Effective triage processes improve efficiency at ED departments by directly impacting length of stay, throughput, and even patient satisfaction.

The Emergency Severity Index (ESI) is a five-level triaging tool that was developed by two ED physicians with the principles of "Who should be seen first?" and "Who can safely wait?"6-9 It works on the basic concept of delivering health care based on the level of urgency. It is a standardized tool used to categorize patients based on their urgency (acuity) and estimated resource consumption. ESI level 1 is considered "emergent," ESI 2 "urgent", and those in ESI 3-5 are stratified based on estimated resource utilization (3 being highest resource consumption and 5 being lowest). ${ }^{5}$

The ESI originated from the US health-care system, and was developed in keeping with local dynamics of case mix and resource utilization in consideration. As its use became prevalent around the world, it became important for other health systems to study this tool in their local context. Various studies have been conducted to establish the trends and usability of the ESI in different parts of the world. ${ }^{10-12}$

The Ministry of Health and Prevention (MOHAP) of the UAE governs a vast health-care network covering over17 hospitals classified into secondary,tertiary, specialized, and community hospitals. These hospitals have EDs in six emirates in the UAE. To keep up with international guidelines and make the triaging process more objective for ED staff, the ESI was introduced gradually as the standard triaging tool in all EDs from 2010.

One of the important catalysts for this initiative was the introduction of an electronic medical record system (Wareed, supplied and implemented by Cerner Corporation) for their EDs, which was acquired by MOHAP in 2009. Within Wareed, the ESI algorithm is part of the standard triage documentation. After performing their triage assessment, nurses can enter the patient's acuity level within Wareed. With an ED-specific module, the electronic medical record system has the capability of capturing all ED visits with relevant data, which can be further utilized for analysis and improvement purposes. The system was introduced after training of all triaging clinicians on the latest ESI guidelines. After implementation, there was a need to revisit the system and evaluate its usability, trends, and impact on the ED-care process.

\section{Aims and Objectives}

This study was conducted to evaluate patterns and trends of population dynamics after implementation of the ESI. Measures under study were:

1. descriptive statistics of the population presenting to EDs during 2018

2. distribution of acuity across different groups (based on age, sex, nationality)

3. average time spent by patients in the ED (length of stay)

4. comparison of assigned acuity to resources consumed by the patients (triage accuracy)

5. association between patient-related factors and triage accuracy (severity of illness, age-group).

\section{Methods}

\section{Design}

This was an observational study conducted retrospectively to ascertain findings from ED data over 1 year (January 2018-December 2018).

\section{Study Setting}

This study was conducted on data collected from 15 hospital EDs operating under MOHAP. These hospitals are distributed among six (out of seven) emirates of the UAE, with a catchment population of approximately 6 million. ${ }^{13}$ Data were captured from all facilities, irrespective of their bed capacity or specialties.

\section{Study Participants}

Since we aimed to study the population dynamics of all patients presenting to MOHAP hospital EDs, all patient records for the study period were extracted.

\section{Data Extraction}

Using suitable techniques and software, all data from EDs of 15 hospitals were extracted. These were all the MOHAP hospitals equipped with the Wareed system in their EDs in 2018. Data included patient demographics (age, sex, and 
nationality), date of visit, acuity assigned by the triage nurse, length of stay, and resources consumed. The ESI algorithm defines a certain intervention as "resources". 6

\section{Resources}

Every resource defined in the ESI handbook was captured as discrete data within the Wareed system. For this study, we defined extensive complex queries (using Structured Query Language) to automate the process of calculating actual resource utilization. As per our literature survey, this was an innovative approach unique for this region. This technique allowed us to investigate a larger data set and observe patterns. The resources included in the resource-utilization checklist were clinical laboratory studies, radiography, electrocardiography monitoring, special studies, fluids, parenteral medications, intramuscular medications, and specialist consultations, as defined in the ESI handbook. ${ }^{6}$

\section{Triage Accuracy}

Triage accuracy was defined as the extent of agreement between standard guidelines and local findings by comparing the suggested resource consumption (for acuity levels 3-5) to the actual resources consumed in these hospitals.

Since acuity levels 1 and 2 are assigned based on patient criticality, rather than expected resource utilization, it is not be possible to validate these without examining the patient. For this reason, we conducted our analysis on patients who had been assigned acuity $3-5$ during this period, while those with 1 and 2 were excluded from this analysis. We also determined the correlation between acuity level and extent of accuracy in terms of resource expenditure.

\section{Data Entry, Collection, and Analysis}

After implementation of the ESI, all data were entered by trained clinicians into Wareed in a standardized format. Data were collected through a logical and standardized process from Wareed, and all required measures were taken to mask patient identity and data storage and ensure safety. Data were analyzed using Microsoft Excel and SPSS 21 to derive statistical inferences. Frequency distributions and correlations were observed among other statistical inferences. We employed the Kruskal-Wallis test of significance and logistic regression methods for further analysis of our data.

This study was conducted by MOHAP and was in line with their ethical policies. It was ethically aligned with the
Declaration of Helsinki. Data were deidentified and all necessary measures taken to maintain privacy and confidentiality of individuals and facilities and to ensure there was minimization of bias.

\section{Results}

A total of 576,154 patients presented to all 15 MOHAP EDs from 1st January 1-December 31, 2018. Facility 15 saw the highest number of patients $(15.5 \%)$ and the fewest were recorded at facility $1(0.4 \%)$. Patient-visit frequency was observed during different months: highest in October (9.5\% of total visits) and lowest in July (7.4\% of the total visits). Patient ages were categorized in nine groups of 10 years' interval each, with the last group including all patients 80 years and above. Most patients were from the youngest age-group of 0-10 years and the fewest from the oldest age-group of 80 years and above. Among all the patients who visited MOHAP EDs in 2018, $54.6 \%$ were male and the rest female. Of these, $61.2 \%$ were from the local (Emirati) population and the rest expatriates (non-Emiratis) Table 1; refer to appendix for tables S1-S7, Figures S1-S4).

Distribution of acuity assigned by triage nurses was analyzed, and it was observed that most patients received an acuity of 4 (42.9\%) very closely followed by acuity 3 $(42.1 \%)$, while the least were assigned an acuity of 1 $(0.3 \%)$. A small number of patients did not have a documented acuity score assigned (1.9\%), and were excluded from the final analysis (Table 1). We also looked at the age and nationality distribution of patients as per the acuity assigned to them. It was observed that for patients aged over 20 years, the highest proportion was for acuity 3 , while patients aged 20 years and below were most frequently assigned 4 . For acuity 3 and 4 , patients from the youngest age-group were the highest contributors, while for acuity 1 patients aged 41-50years were the highest contributors.

The nationality distribution of acuity was analyzed after dividing the population in two discrete groups of local and non-local populations. We found that for higher acuity (1-3) the major proportion of patients were non-locals, but this trend reversed for acuity 4 and 5 (Table 2). Median length of stay (in minutes) was calculated for all encounters, and after eliminating missing and erroneous entries it was found that overall median length of stay for all patients was 78 minutes. Length of stay was significantly higher for higher severity of illness, as it displayed a decreasing trend from acuity 1 (171 minutes) to 5 (52 
Table I Population Dynamics

\begin{tabular}{|c|c|c|}
\hline & $\mathbf{n}$ & Percentage \\
\hline \multicolumn{3}{|c|}{ Age-group (years) } \\
\hline $0-10$ & 153,804 & 26.7 \\
\hline $11-20$ & 73,665 & 12.8 \\
\hline $21-30$ & 122,998 & 21.3 \\
\hline $31-40$ & 101,046 & 17.5 \\
\hline $4 I-50$ & 48,571 & 8.4 \\
\hline $5 I-60$ & 31,147 & 5.4 \\
\hline $61-70$ & 22,235 & 3.9 \\
\hline $7 I-80$ & $|2,8| 2$ & 2.2 \\
\hline $80+$ & 7,213 & 1.3 \\
\hline Missing & 2,663 & 0.5 \\
\hline \multicolumn{3}{|l|}{ Sex } \\
\hline Female & 261,500 & 45.4 \\
\hline Male & 314,637 & 54.6 \\
\hline Missing & 17 & 0.0 \\
\hline \multicolumn{3}{|l|}{ Nationality } \\
\hline Emirati & 352,513 & 61.2 \\
\hline Non -Emirati & $223,64 I$ & 38.8 \\
\hline \multicolumn{3}{|l|}{ Acuity } \\
\hline I & 1,948 & 0.3 \\
\hline 2 & 21,044 & 3.7 \\
\hline 3 & 242,538 & 42.1 \\
\hline 4 & 247,365 & 42.9 \\
\hline 5 & $52,|8|$ & 9.1 \\
\hline Missing & $1 \mathrm{I}, 078$ & 1.9 \\
\hline Total & 576,154 & 100 \\
\hline
\end{tabular}

minutes). This difference in medians was analyzed for significance using the Kruskal-Wallis test of significance and found to be statistically significant ( $p=0$, Figure 1).
A rigorous method of comparing resources utilized by the patient versus the acuity assigned ${ }^{2-14}$ by the triaging clinician was performed to assess the accuracy of triage. We found that for $41.6 \%$ of visits, there was an agreement between the resources estimated and those utilized in the ED. Among the mismatched cases, the majority were overtriaged into a higher category $(79.5 \%)$, while the rest were undertriaged. Since this matching was done utilizing the resource consumption of the patients, it was not possible to conduct this evaluation for acuity levels 1 and 2 . It was not possible to assign a patient an acuity of 1 or 2 based on resource consumption, so there were no cases of undertriage in acuity 3. The reverse situation was seen with acuity 5, where there were no cases of over-triaging (Tables 3 and 4).

We applied logistic regression to ascertain the relationship between certain institutional and patient factors with triage accuracy. While results for some factors were not statistically significant (sex, nationality, month of arrival, hospital-bed capacity, hospital footfall), we found a positive association between severity of illness and accuracy of triage (OR $0.14, p=0)$. There was a significant correlation between patient age-group and accuracy of triage as well, where those aged 11-20 years had the highest probability of accurate triage acuity $\left(R^{2}=0.41, p=0\right.$; Table 5$)$.

\section{Discussion}

Our study is an attempt to understand the dynamics of the population utilizing ED services and utilization trends of an ED-triage tool (ESI) in the UAE. Since this study was

Table 2 Acuity-Assigned Cross-Tabulations Against Age-Group and Nationality

\begin{tabular}{|c|c|c|c|c|c|c|c|}
\hline Acuity & $\mathbf{I}$ & 2 & 3 & 4 & 5 & Missing & Total \\
\hline \multicolumn{8}{|c|}{ Age-group, years } \\
\hline $0-10$ & 182 & 3,348 & 56,558 & 74,282 & 16,888 & 2,546 & 153,804 \\
\hline $11-20$ & 85 & 1,050 & 20,652 & 42,316 & 8,765 & 797 & 73,665 \\
\hline $21-30$ & 246 & 4,175 & 53,124 & 51,990 & 11,267 & 2,196 & 122,998 \\
\hline $31-40$ & 299 & 4,123 & 48,310 & 38,348 & 8,061 & I,905 & 101,046 \\
\hline $4 I-50$ & 313 & 2,836 & 25,633 & 16,179 & 2,897 & 714 & 48,572 \\
\hline $5 I-60$ & 292 & 2,205 & 16,478 & 9,957 & $|, 75|$ & 464 & 31,147 \\
\hline $6 I-70$ & 262 & $\mathrm{I}, 566$ & 11,239 & 7,399 & 1,299 & 471 & 22,236 \\
\hline $7 I-80$ & 152 & I,099 & 6,731 & 3,963 & 573 & 294 & 12,812 \\
\hline $80+$ & 117 & 637 & 3,731 & 2,216 & 307 & 205 & 7,213 \\
\hline Missing & 0 & 5 & 82 & 715 & 373 & $\mathrm{I}, 486$ & 2,661 \\
\hline Total & 1,948 & 21,044 & 242,538 & 247,365 & 52,181 & $\mathrm{II}, 078$ & 576,154 \\
\hline \multicolumn{8}{|l|}{ Nationality } \\
\hline Emirati & 410 & 6,814 & 91,061 & 203,37| & 42,752 & 8,105 & 352,513 \\
\hline Non-Emirati & $\mathrm{I}, 538$ & 14,230 & $|5|, 477$ & 43,994 & 9,429 & 2,973 & 223,641 \\
\hline
\end{tabular}




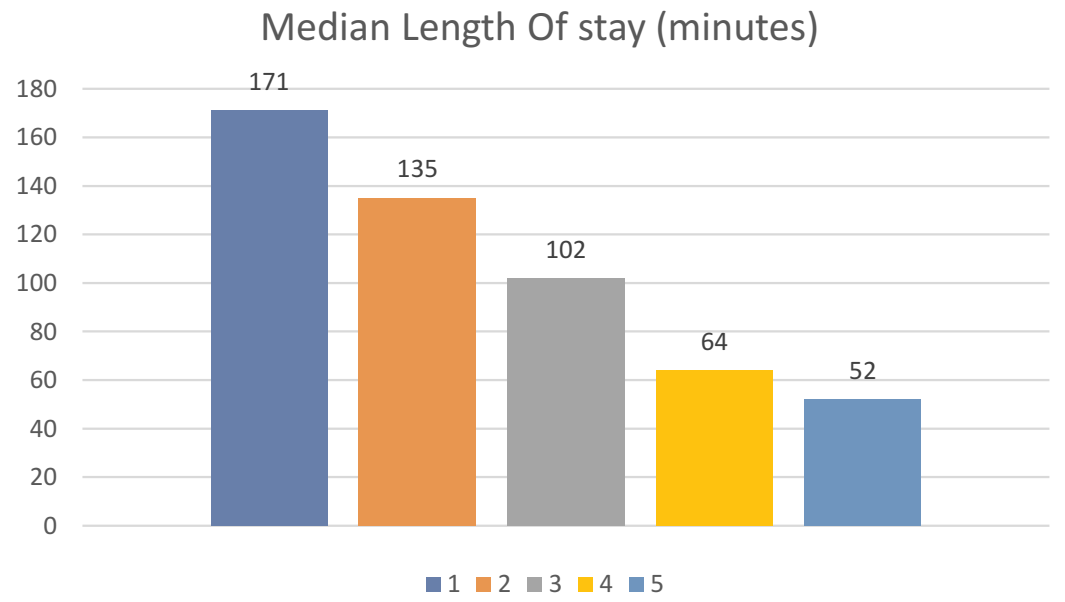

Figure I Median length of stay for different acuity levels. I:Acuity I,2: Acuity 2, 3: Acuity 3, 4:Acuity 4,5:Acuity 5

conducted on the entire population presenting to EDs in 2018 , it is truly reflective of population trends. The trends of ED visits were similar to those observed in other parts of the world. Though there was no major difference, the greatest traffic in EDs was observed in October and the lowest in July. This finding is consistent with findings from other studies, ${ }^{15,16}$ and explains seasonal and population dynamics. Sex distribution was also similar to other studies, where more male patients attend the ED than female patients. ${ }^{17}$. The most frequent visitors were those

Table 3 Agreement Between Assigned Acuity (3-5),and Triage Accuracy(based on Resources Consumed)

\begin{tabular}{|l|l|l|}
\hline \multicolumn{1}{|c|}{} & $\mathbf{n}$ & Percentage \\
\hline $\begin{array}{l}\text { Acuity agreement } \\
\text { Matched }\end{array}$ & & \\
Unmatched & 225,386 & 41.6 \\
Total & 316,698 & 58.4 \\
\hline Unmatched acuity & 542,084 & 100 \\
Over-triage & & \\
Under-triage & 251,933 & 79.5 \\
Total & 64,765 & 20.5 \\
\hline
\end{tabular}

in the youngest age group (0-10) followed by those in the third (21-30) age group, while the least frequent were elderly. According to the population pyramid of the $\mathrm{UAE}^{18}$ the highest population concentration is aged 30-40 years and those older than 80 years are much less.

Trends in service utilization between local and nonlocal users were studied to understand the impact of policies on utilization behavior. In the MOHAP hospitals, any patient who visits the ED with an acuity of 1-3 is not charged for the visit, but they must pay a consultation fee if their acuity is 4 or 5 . The intention of this policy is to discourage ED use for simpler ailments and divert traffic toward primary-care facilities. The local Emirati population is exempt from this additional charge and hence provides a perfect control group to study the impact of this policy. We observed that among patients with acuity $1-3$, the percentage of non-locals was higher than acuity 4 and 5, where locals utilized ED services more. These data demonstrate that non-locals avoid ED visits unless the condition is relatively critical/urgent. However, there was no significant impact of nationality on the level of triage accuracy, which reflects on the unbiased practices of the clinicians.

Table 4 Cross-Tabulation: Acuity and Unmatched Triage

\begin{tabular}{|l|l|l|l|l|l|}
\hline \multirow{2}{*}{ Acuity Assigned } & \multicolumn{2}{|l|}{ Over-triage } & \multicolumn{2}{l|}{ Under-triage } & \multirow{2}{*}{ Total } \\
\cline { 2 - 5 } & $\mathbf{n}$ & Percentage & $\mathbf{n}$ & Percentage & \\
\hline 3 & 104,761 & 100 & 0 & 0 & 104,761 \\
4 & 147,172 & 72.7 & 55,302 & 27.3 & 202,474 \\
5 & 0 & 0 & 9463 & 100 & 9,463 \\
\hline
\end{tabular}


Table 5 Multivariate Logistic Regression Analysis for Association of Triage Accuracy with Independent Patient Factors

\begin{tabular}{|l|l|l|l|}
\hline & $\boldsymbol{\beta}$ (exponential) & $\boldsymbol{P}$ & $\boldsymbol{R}^{2}$ \\
\hline Acuity level & & & \\
3 & $\mathrm{I}$ & 0 & 0.14 \\
4 & 0.274 & 0 & \\
5 & 0.178 & & \\
\hline Age-group, years & & & $0.4 \mathrm{I}$ \\
$0-10$ & $\mathrm{I}$ & 0 & \\
II-20 & 0.053 & 0 & \\
$2 \mathrm{I}-30$ & -0.063 & 0 & \\
$3 \mathrm{I}-40$ & -0.117 & 0 & \\
$4 \mathrm{I}-50$ & -0.099 & 0 & \\
$5 \mathrm{I}-60$ & -0.107 & 0 & \\
$6 \mathrm{I}-70$ & -0.087 & 0 & \\
$7 \mathrm{I}-80$ & -0.080 & 0 & \\
$80+$ & -0.063 & \\
\hline
\end{tabular}

It was observed that length of stay was proportional to seriousness of illness assigned to a patient. As acuity went from 5 toward 1, median length of stay increased significantly. Many other studies have analyzed this relationship and found a positive relationship between higher acuity and increased length of stay in the ED. ${ }^{19-22}$ This is explained by the fact that patients with higher acuity are more critical and require more resources and time to stabilize before a decision of admission/discharge can be taken. Several models have been proposed to reduce this length of stay and hence reducing ED crowding. We propose a further analysis of all factors (including resource consumption) contributing to increased length of stay in all patients and appropriate treatment paths according to ESI category. Other studies have conducted such analyses and proposed separate ED paths for urgent versus nonurgent patients. . $^{19,23,24}$

Even though the ESI was established in a different setting, we were able to find results that were coherent with guideline expectations. According to other research, $40 \%$ of ED patients are expected to be in acuity 3 , and we found this to $42.1 \%{ }^{3,9}$ Some studies conducted locally have quoted accuracy of prediction of required resources by experienced nurses beginning as high as $58.7 \%$. These studies were conducted using 25 standard cases presented in the ESI handbook. In contrast, our analysis was done on more than half a million cases as a retrospective study using automated methods for data capture and analysis, but we were restricted to analyzing accuracy on triage levels $3-5{ }^{25}$

Before introduction of the ESI triaging system, all ED clinicians were trained on up-to-date guidelines to ensure proper understanding of assigned acuity (according to clinical status) with the extent of resources the patient would consume during their stay. After vigorous analysis, matching between acuity assigned and total resources consumed during ED stay was assessed to gauge the accuracy of triage. If this was in line with ESI guidelines, ${ }^{3}$ it was taken as the correct allocation, but if the assigned acuity did not match the resources consumed, it was categorized as either over- or undertriage. Correct acuity assignment plays a pivotal role in defining ED-treatment module, and incorrect assignment can lead to unnecessary resource consumption and possible increased waiting time for other patients. ${ }^{26-29}$ There have been mixed results from various studies to have observed this level of agreement. We found that $41.6 \%$ of our patients received accurate triage acuity, whereas the rest were not in agreement. This variation could have been due to various factors, which calls for further in-depth analysis to uncover them.

Further detailed analysis should look into the accuracy of the triage checklist (eg, vitals measurement, pain scoring, and comorbid conditions). Literature supports the role of nursing experience and retraining/reinforcement sessions in improved performance on triage. ${ }^{30,31}$ A learning-needs assessment based on ED nurses' working experience, knowledge-base assessment, and learning competence can give an in-depth understanding of any nursing-related factors, creating an impact on accuracy of triage assignment. This might also be an indication of a reinforcement of nursing training, where the before and after mistriage rate can be compared to evaluate refresher-training needs.

We were able to find a significant correlation between severity of illness and accuracy of triage. We also found that patients in certain age-groups had a higher chance of receiving accurate triage than others. Our findings are in line with several other studies that indicated that patients with higher acuity are mostly more accurately triaged by the triage clinician. ${ }^{32-34}$ Studies conducted on correlation between age and triage accuracy have exhibited mixed results, with some stating that the accuracy of triage is lower in higher age-groups, ${ }^{35}$ while some studies have stated the opposite. ${ }^{36}$

\section{Strengths}

We present a large data cohort representing six emirates of the UAE. The study was composed of a large case mix, with all ages, nationalities, and all-year data. According to 
the literature, the public health sector caters to a larger population than the private sector. ${ }^{37}$ Our study was done retrospectively, eliminating the possibility of Hawthorne bias. These factors make our study relevant and reliable in the UAE context.

\section{Limitations}

Since this analysis was conducted retrospectively, we could not assess accuracy of acuity 1 and 2 . The most recommended methods for validating the aforementioned are through inter-rater validity assessment, ${ }^{38,39}$ which was not possible in our retrospective analysis. Private health-sector data were not included, which might be a preferred option for the non-local population. It is clear from our data that less critical non-local patients do not resort to public facilities. There might be an impact of the patient's socioeconomic status on service preference and urgency of care, ${ }^{40}$ but this was beyond the scope of our study.

\section{Conclusion}

Our study is the first of its kind in the UAE region, and was conducted on a very large population from the country. It reflects on ED-utilization trends and dynamics for a very large population cohort residing in the UAE. We found a statistically significant increase in length of stay with increasing severity of illness. This can lead to further analysis of the types of illness and introduction of specific fast tracks for early admission of these patients. We found that the accuracy of triage was higher for more serious patients and certain age-groups. We propose further studies and targeted interventions to address those groups with lower accuracy and to scale up this practice further.

\section{Funding}

This research did not receive any specific grant from funding agencies in the public, commercial, or not-forprofit sectors.

\section{Disclosure}

This is a joint paper of the Ministry of Health and Prevention UAE and Cerner Middle East. The authors report no potential conflicts of interest for this work.

\section{References}

1. Ebrahimi M, Mirhaghi A, Mazlom R, et al. The role descriptions of triage nurse in emergency department: a Delphi Study. Scientifica. 2016;2016:1-6. doi:10.1155/2016/5269815

2. Mirhaghi A, Ebrahimi M, Heydari A, et al. The reliability of the Canadian triage and acuity scale: meta-analysis. $N$ Am J Med Sci. 2015;7(7):299. doi:10.4103/1947-2714.161243

3. Cioffi J. Decision making by emergency nurses in triage assessments. Accid Emerg Nurs. 1998;6(4):184-191. doi:10.1016/S0965-2302(98)90077-7

4. Fry M, Burr G. Current triage practice and influences affecting clinical decision-making in emergency departments in NSW, Australia. Accid Emerg Nurs. 2001;9(4):227-234. doi:10.1054/ aaen.2001.0268

5. Dong S. The need for reliable and valid triage. Acad Emerg Med. 2005;12(10):1013. doi:10.1197/j.aem.2005.07.002

6. Emergency severity index, version 4: everything you need to know, May 2005, (DVD). [Place of publication not identified]:: [publisher not identified]. 2011.

7. Gilboy N. The Emergency Severity Index. Rockville, MD: U.S. Dept. of Health and Human Services, Public Health Service, Agency for Healthcare Research and Quality; 2005.

8. Shelton R. The emergency severity index 5-level triage system. Dimens Crit Care Nurs. 2009;28(1):9-12. doi:10.1097/01. dcc.0000325106.28851.89

9. Travers D, Waller A, Bowling J, et al. Five-level triage system more effective than three-level in tertiary emergency department. $J$ Emerg Nurs. 2002;28(5):395-400. doi:10.1067/men.2002.127184

10. Eitel D. The emergency severity index triage algorithm version 2 is reliable and valid. Acad Emerg Med. 2003;10(10):1070-1080. doi:10.1197/s1069-6563(03)00350-6

11. Eitel D, Gilboy N, Rosenau A, et al. Does this patient meet the criteria for emergency severity index level 2? J Emerg Nurs. 2008;34(4):382-383. doi:10.1016/j.jen.2008.04.027

12. van der Wulp I, Schrijvers A, van Stel H. Predicting admission and mortality with the emergency severity index and the manchester triage system: a retrospective observational study. Emerg Med J. 2009;26(7):506-509. doi:10.1136/emj.2008.063768

13. United Arab Emirates Population Statistics (2020). Official GMI Blog. 2020. Available from: https://www.globalmediainsight.com/ blog/uae-population-statistics/. Accessed October 15, 2020.

14. Gerdtz M, Bucknall T. Triage nurses' clinical decision making. An observational study of urgency assessment. J Adv Nurs. 2001;35 (4):550-561. doi:10.1046/j.1365-2648.2001.01871.x

15. Gulacti U, Lok U, Celik M, et al. The ED use and non-urgent visits of elderly patients. Turk $J$ Emerg Med. 2016;16(4):141-145. doi:10.1016/j.tjem.2016.08.004

16. Emergency Department Visits A report to the Maricopa County Community from Arizona Health Query a Community - University Partnership. Available from: https://chs.asu.edu/sites/default/files/pub lication_28.pdf. Accessed March 9, 2020.

17. Ko M, Lee Y, Chen C, et al. Prevalence of and predictors for frequent utilization of emergency department. Medicine. 2015;94(29):e1205. doi: $10.1097 / \mathrm{md} .000000000001205$

18. 5 Populationpyramid.net. 2020. Available from: https://www.population pyramid.net/united-arab-emirates/2019/. Accessed November 10, 2019.

19. Arya R, Wei G, McCoy J, et al. Decreasing length of stay in the emergency department with a split emergency severity index 3 patient flow model. Acad Emerg Med. 2013;20(11):1171-1179. doi:10.1111/acem.12249

20. Lauks J, Mramor B, Baumgartl K, et al. Medical team evaluation: effect on emergency department waiting time and length of stay. PLoS One. 2016;11(4):e0154372. doi:10.1371/journal. pone. 0154372 
21. Chaou C, Chiu T, Yen A, et al. Analyzing factors affecting emergency department length of stay-using a competing risk-accelerated failure time model. Medicine. 2016;95(14):e3263. doi:10.1097/ md.0000000000003263

22. Chaou C, Chen H, Chang S, et al. Predicting length of stay among patients discharged from the emergency department-using an accelerated failure time model. PLoS One. 2017;12(1):e0165756. doi:10.1371/journal.pone.0165756

23. Asaro P, Lewis L, Boxerman S. The impact of input and output factors on emergency department throughput. Acad Emerg Med. 2007;14(3):235-242. doi:10.1197/j.aem.2006.10.104

24. Adopt This 5-Part Process to Reduce ER Length of Stay | HealthLeaders Media. Healthleadersmedia.com. 2020. Available from: https://www.healthleadersmedia.com/clinical-care/adopt5-part-process-reduce-er-length-stay. Accessed November 12, 2019.

25. Mistry B, Hinson J, Balhara K, et al. 165 assessing accuracy and inter-rater reliability of the emergency severity index in triage in the Al-Rahba emergency department: a Cross-Sectional Observational Study. Ann Emerg Med. 2016;68(4):S65. doi:10.1016/j.annemergmed. 2016.08.178

26. Andrulis D, Kellermann A, Hintz E, et al. Emergency departments and crowding in United States teaching hospitals. Ann Emerg Med. 1991;20(9):980-986. doi:10.1016/s0196-0644(05)82976-2

27. Bazarian J, Schneider S, Newman V, et al. Do admitted patients held in the emergency department impact the throughput of treat-andrelease patients? Acad Emerg Med. 1996;3(12):1113-1118. doi:10.1111/j.1553-2712.1996.tb03370.x

28. Chi C, Huang C. Comparison of the Emergency Severity Index (ESI) and the Taiwan Triage System in predicting resource utilization. J Formos Med Assoc. 2006;105(8):617-625. doi:10.1016/s09296646(09)60160-1

29. Wuerz R, Milne L, Eitel D, et al. Reliability and validity of a new five-level triage instrument. Acad Emerg Med. 2000;7(3):236-242. doi:10.1111/j.1553-2712.2000.tb01066.x

30. Brosinski C, Riddell A, Valdez S. Improving triage accuracy. Clin Nurse Spec. 2017;31(3):145-148. doi:10.1097/nur.000000000000 0291
31. Soontorn T, Sitthimongkol Y, Thosingha O, Viwatwongkasem C. Factors influencing the accuracy of triage by registered nurses in Trauma patients. Pac Rim Int J Nurs Res. 2018;22(2):120-129.

32. Mirhaghi A. Outcomes for emergency severity index triage implementation in the emergency department. J Clin Diagn Res. 2015. doi:10.7860/jcdr/21015/11791.5737

33. Grossmann F, Nickel C, Christ M, et al. Transporting clinical tools to new settings: cultural adaptation and validation of the emergency severity index in German. Ann Emerg Med. 2011;57(3):257-264. doi:10.1016/j.annemergmed.2010.07.021

34. Tanabe P, Gimbel R, Yarnold P, et al. Reliability and validity of scores on the emergency severity index version 3. Acad Emerg Med. 2004;11(1):59-65. doi:10.1111/j.1553-2712.2004.tb01371.x

35. Arslanian-Engoren $C$. Gender and age bias in triage decisions. $J$ Emerg Nurs. 2000;26(2):117-124. doi:10.1016/s0099-1767(00) 90053-9

36. Platts-Mills T, Travers D, Biese K, et al. Accuracy of the emergency severity index triage instrument for identifying elder emergency department patients receiving an immediate life-saving intervention. Acad Emerg Med. 2010;17(3):238-243. doi:10.1111/j.1553-2712.20 10.00670.x

37. Apps.who.int. 2019. Available from: http://apps.who.int/medicine docs/documents/s17313e/s17313e.pdf. Accessed November 25, 2019.

38. Mistry B, Stewart De Ramirez S, Kelen G, et al. Accuracy and reliability of emergency department triage using the emergency severity index: an international multicenter assessment. Ann Emerg Med. 2018;71(5):581-587.e3. doi:10.1016/j.annemergmed.2017.09. 036

39. Baumann M. Evaluation of the emergency severity index (version 3) triage algorithm in pediatric patients. Acad Emerg Med. 2005;12 (3):219-224. doi:10.1197/j.aem.2004.09.023

40. Khan Y, Glazier R, Moineddin R, et al. A population-based study of the association between socioeconomic status and emergency department utilization in Ontario, Canada. Acad Emerg Med. 2011;18 (8):836-843. doi:10.1111/j.1553-2712.2011.01127.x

\section{Publish your work in this journal}

The Open Access Emergency Medicine is an international, peerreviewed, open access journal publishing original research, reports, editorials, reviews and commentaries on all aspects of emergency medicine. The manuscript management system is completely online and includes a very quick and fair peer-review system, which is all easy to use. Visit http://www.dovepress.com/testimonials.php to read real quotes from published authors. 